\title{
SUPORTE NUTRICIONAL NO PACIENTE IDOSO: DEFINIÇÃO, DIAGNÓSTICO, AVALIAÇÃO E INTERVENÇÃO
}

NUTRITIONAL SUPPORT IN GERIATRIC PATIENTS:

DEFINITION, DIAGNOSIS, ASSESSMENT AND THERAPEUTIC

Júlio Sérgio Marchini' ${ }^{1,2}$; Eduardo Ferriolli² \& Julio Cesar Moriguti ${ }^{2}$

'Docente da Divisão de Nutrição Clínica; ${ }^{2}$ Médico Assistente da Divisão de Clínica Médica Geral e Geriatria. Departamento de Clínica Médica da Faculdade de Medicina de Ribeirão Preto da Universidade de São Paulo.

CorRespondência: Prof. Dr. Júlio Sérgio Marchini - Divisão de Nutrição Clínica do Departamento de Clínica Médica da Faculdade de Medicina de Ribeirão Preto - CEP: 14049-900 - Ribeirão Preto - SP; Email: jsmarchi@fmrp.usp.br

MARCHINI JS; FERRIOLI E \& MORIGUTI JC. Suporte nutricional no paciente idoso: definição, diagnóstico, avaliação e intervenção. Medicina, Ribeirão Preto, 31: 54-61, jan./mar. 1998.

RESUMO: Atualmente, vem ocorrendo um aumento importante no número de pacientes idosos submetidos a internação hospitalar, mantidos em casas de repouso ou atendidos em regime ambulatorial, cujo estado nutricional pode ser considerado crítico. Assim, é fundamental que as alterações próprias do envelhecimento sejam o mais precocemente possível diferenciadas dos sinais clínicos de desnutrição. Um alto grau de suspeita clínica de pacientes idosos desnutridos, e que, conseqüentemente, necessitam de terapêutica nutricional, pode ser obtido por meio da história clínica, exame físico e dados laboratorias apropriados. A má nutrição que ocorre no idoso pode ser devida às alterações fisiológicas do envelhecimento, às condições sócio-econômicas, às doenças e à interação entre nutrientes e medicamentos. Assim sendo, as principais causas de má nutrição podem ser catalogadas como secundárias ao envelhecimento, menor rendimento econômico, isolamento, a morte de entes queridos, doenças e outros fatores relacionados. Como resultado, o idoso apresenta sério comprometimento do estado geral e uma maior morbidade e mortalidade em geral. A intervenção nutricional utiliza nutrientes, como fármacos, visando o tratamento de doenças. Por fim, as recomendações têm por objetivo indicar a quantidade mínima de nutrientes que seria adequada para a maioria das pessoas em seu ambiente usual, sem traumas ou doenças. No entanto, as recomendações, para o idoso são extrapoladas das recomendações obtidas para crianças e adultos jovens, nem sempre próprias para o idoso.

UNITERMOS: Nutrição. Idoso. Dietoterapia.

\section{INTRODUÇÃO}

Atualmente, vem ocorrendo um aumento importante no número de pacientes idosos submetidos a internação hospitalar ${ }^{(1)}$, mantidos em casas de repouso ${ }^{(2)}$ ou seguidos em regime ambulatorial ${ }^{(3)}$, cujo estado nutricional pode ser considerado crítico $^{(4)}$. No Hospital das Clínicas da Faculdade de Medicina de Ribeirão Preto, a ocorrência desse fenômeno é nítida: o número de internações de pessoas jovens vem diminuindo; em contraposição, o número de idosos internados vem aumentando significativamente, Tabela I. A proporção de internações entre as diferentes faixas etárias, também se alterou nitidamente, entre 1980 e 1995, apontando para o número crescente de idosos, Figura 1. Ao médico, cabe o desafio de estabelecer um plano terapêutico, visando a recuperação e reabilitação desses pacientes, cujo tratamento é freqüentemente multidimensional. Independente da causa da internação, em pacientes com mais de sessenta anos, existe uma correlação significativa entre os menores valores da área muscular do braço (< quinto percentil), perda de peso ( $>20 \%$ do peso habitual), hipoprealbuminemia $(<150 \mathrm{mg} / \mathrm{dl})$ e a maior mortalidade, tanto no nível intra-hospitalar quanto no do domícilio ${ }^{(5)}$. 
Tabela I - Número de internações no Hospital das Clínicas da Faculdade de Medicina de Ribeirão Preto, de acordo com a faixa etária, nos anos de 1980 e 1995

\begin{tabular}{lrrr}
\hline Faixa etária & 1980 & 1995 & Variação \% \\
\hline $0-10$ & 4117 & 2830 & -31 \\
$11-20$ & 1979 & 1548 & -22 \\
$21-30$ & 3179 & 1886 & -41 \\
$31-40$ & 1897 & 2236 & +18 \\
$41-50$ & 1300 & 2024 & +56 \\
$51-60$ & 915 & 1854 & +103 \\
$61-70$ & 734 & 1784 & +143 \\
$71-80$ & 316 & 1022 & +223 \\
$81+$ & 116 & 286 & +147 \\
\hline
\end{tabular}
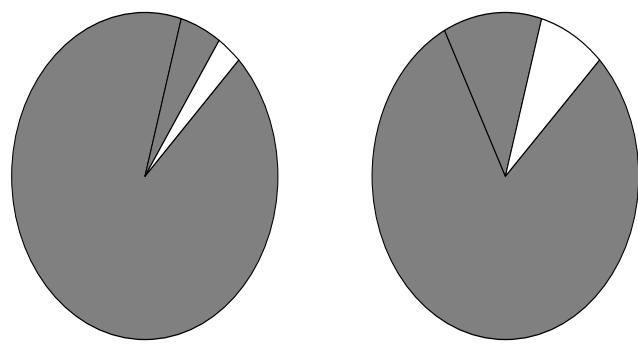

$\square$

$\square$

$\square$

Figura 1 - Proporção de pacientes internados no Hospital das Clínicas de Ribeirão Preto, nos anos de 1980 e 1995, por faixa etária em anos.

Existem evidências de que cerca de $70 \%$ dos idosos institucionalizados ingerem dieta deficiente em energia e fibras ${ }^{(6)}$, e entre os pacientes idosos internados, cerca de $80 \%$, apresentam níveis reduzidos de albuminemia e 50\%, emagrecimento importante ${ }^{(7)}$. Em contraste a esses dados, os idosos (73-90 anos) da Finlândia, que vivem em suas casas, têm maior probabilidade de apresentarem ingestão alimentar e medidas antropométricas adequadas, a não ser pela baixa ingestão de zinco e ácido fólico ${ }^{(8)}$. Em Israel $^{(9)}$, entre os idosos que residem em casa própria, menos de $17 \%$ apresentam albuminemia menor que $3,5 \mathrm{~g} / \mathrm{dl} \mathrm{e}$ menos de $2 \%$ têm índice de massa corporal menor que $19 \mathrm{~kg} / \mathrm{m}^{2}$. Na Irlanda, idosos, a nível domiciliar, com mais de noventa anos têm valores de índice de massa corporal em torno de $24 \mathrm{~kg} / \mathrm{m}^{2}$ e prega cutânea tricipital de $12 \mathrm{~mm}$, medidas estas considerados adequadas ${ }^{(10)}$. Com o avançar da idade, existe um aumento progressivo da quantidade de tecido adiposo.

Assim, é fundamental que as alterações próprias do envelhecimento sejam o mais precocemente possível diferenciadas dos sinais clínicos de desnutrição. Um alto grau de suspeita clínica de pacientes idosos desnutridos, e que, conseqüentemente, necessitam de terapêutica nutricional, pode ser obtido por meio da história clínica, exame físico e dados laboratorias apropri$\operatorname{ados}^{(11)}$. A terapêutica nutricional desempenha papel importante na promoção da saúde, prevenção da doença e no cuidado geral, tanto em situações clínicas como em cirúrgicas ${ }^{(12)}$.

Uma das maneiras de avaliar o estado nutricional seria a procura de indicadores que estivessem mais relacionados com uma maior morbidade e/ou mortalidade. Pacientes idosos, portadores de desnutrição proteicocalórica, apresentam risco aumentado de adquirir doenças e/ou de evoluir de forma desfavorável a um tratamento específico.

\section{MÁ NUTRIÇÃO NO IDOSO}

Os processos carenciais no idoso, em analogia a outros grupos etários, podem ser subdivididos, didaticamente, em quatro grupos, conforme mostrado na Tabela II.

A má nutrição protéica, também identificada como kwashiorkor, é caraterizada por índices antropométricos praticamente inalterados devido ao edema ou, até mesmo, pelo acúmulo de tecido adiposo e bioquímica nutricional, apresentando valores abaixo do esperado. Assim, não é raro encontrar pacientes em anasarca, com peso mantido e albuminemia de $1.5 \mathrm{mg} / \mathrm{dl}$.
Tabela II - Classificação dos processos carências que envolvem os idosos

\begin{tabular}{lcc}
\hline Má nutrição & $\begin{array}{c}\text { Dados } \\
\text { antropométricos }\end{array}$ & $\begin{array}{c}\text { Bioquimica } \\
\text { nutricional }\end{array}$ \\
\hline Calórica - Marasmo & $\downarrow \downarrow$ & $\leftrightarrow$ \\
Protéica - "Kwashiorkor" & $\leftrightarrow$ & $\downarrow \downarrow$ \\
Proteicocalórica & $\downarrow \downarrow$ & $\downarrow \downarrow$ \\
Específica & $\leftrightarrow$ & $\downarrow \downarrow$ ou $\uparrow \uparrow$
\end{tabular}


Os pacientes portadores de má nutrição calórica, ou energética, também identificada como marasmo, apresentam medidas antropométricas, muito abaixo do padronizado, e manutenção, em limite inferior, dos valores de bioquímica nutricional. Nesses casos, ocorre deficiência da ingestão energética. A resposta imune também se encontra comprometida.

Uma diminuição acentuada das medidas antropométricas e bioquímicas caracteriza os casos de má nutrição proteicocalórica, além de compromentimento imunológico importante. Nesses casos, há uma deficiência global da ingestão de nutrientes.

A má nutrição específica se caracteriza pela deficiência isolada ou múltipla de vitaminas, de minerais ou de oligoelementos. Pode ocorrer isolada ou associada à má nutrição calórica e/ou protéica.

\subsection{Ingestão alimentar}

A ingestão de alimentos, em geral, pode ser diretamente relacionada com estados mórbidos de pacientes idosos Tabela III. Quanto menor for a ingestão calórica, por ocasião da internação, mais previsível será que os pacientes sejam mais velhos, tenham peso menor, maior taxa de dependência funcional e maior morbidade, medida pela maior presença de doenças associadas ${ }^{(13)}$. Pode-se afirmar que a má nutrição do idoso se correlaciona, positivamente e independentemente, com períodos de internações mais longos e maior mortalidade ${ }^{(14)}$.

\subsection{Anorexia}

A perda de peso do idoso, freqüentemente, vem associada à anorexia, sendo esta, muitas vezes, diagnosticada como a causa da perda de peso. Esta anorexia está associada a um maior efeito inibidor do apetite, provocado pela colecistoquinina, menor efeito de opióides e do óxido nitrico ${ }^{(15)}$. Uma variedade de condições sociais, psicológicas e médicas também resultam em anorexia patológica, entre elas, a síndrome depressiva, neoplasias e artrite reumatóide. A anorexia dita senil pode estar associada à síndrome depen- dente de citocinas ${ }^{(15)}$. Além de um suporte nutricional, agressivo, tanto via enteral como parenteral, várias drogas (hormônio do crescimento, megestrol, "ciprohepatadine", tetraidrocanabinol, esteróides anabolizantes e antidepressivos) têm sido utilizadas na tentativa de tratar a anorexia do idoso ${ }^{(15)}$.

Em particular, nos casos de demência, não existe ainda um consenso de qual seria o melhor suporte nutricional. Ao que se sugere, a nutrição enteral, por meio de sonda naso-entérica, não resulta em benefício ou melhora de qualidade de vida para tais pacientes ${ }^{(16)}$.

\subsection{Suplementação de vitaminas e de ferro}

A suplementação de vitaminas hidrossolúveis (50\% do $\mathrm{RDA}^{(17)}$ ), adicionada em $50 \mathrm{~g}$ de açúcar, resulta em melhora significativa dos níveis de tiamina, piridoxina, vitamina $\mathrm{C}$ e ganho de peso, em pessoas com mais de sessenta e cinco anos ${ }^{(18)}$. Recomenda-se que a oferta de vitamina $C$ seja de, no mínimo, $150 \mathrm{mg} /$ dia em sujeitos com mais de oitenta anos. Para a riboflavina e vitamina $\mathrm{B}_{6}$, as recomendações seriam de, no mínimo, $3 \mathrm{mg} / \mathrm{dia}^{(19)}$.

As recomendações de 10-11 mg/dia de ferro não são suficientes para impedir que a prevalência de anemia, em idosos, seja consideravelmente alta ${ }^{(20)}$.

\subsection{Osteoporose}

Fragilidade óssea, resultante da osteoporose, acomete significante número de mulheres idosas e outros pacientes de risco. Os principais fatores de risco incluem os estados hipogonadais (particularmente no período pós-menopausa), fumo, baixa ingestão de cálcio, sedentarismo, história familiar e o uso de certas medicações. A estratégia de prevenção da osteoporose é baseada na manutenção da massa óssea pela dieta, exercício, uso apropriado da terapêutica de reposição hormonal e eliminação de fatores de risco, como o fumo e certos medicamentos (por exemplo, corticóides). Muitos agentes famacológicos para o tratamento da osteoporose estão sendo examinados. Entre eles, incluem-se o alendronato e a calcitonina. No entanto, o uso de fárma-

Tabela III - Características de pacientes idosos por ocasião da internação, ingestão alimentar e morbidade ${ }^{(13)}$

\begin{tabular}{lccr}
\hline Ingestão calórica & $<40 \%$ da recomendada & $>40 \%$ da recomendada & $\mathrm{p}<$ \\
\hline Idade (média $\pm \mathrm{SD}$ ) anos & $80 \pm 7$ & $77 \pm 5$ & 0.05 \\
Dependência funcional \% & 22 & 15 & 0.001 \\
Índice de massa corporal kg/m² & $23 \pm 5$ & $25 \pm 4$ & 0.004 \\
Morbidade \% presença de outras doenças & $4 \pm 2$ & $3 \pm 2$ & 0.02 \\
\hline
\end{tabular}


cos pressupõe indicação médica, correta, além de programa nutricional e de exercício físico, apropriados ${ }^{(21)}$.

\subsection{Suplementação calórica}

Sujeitos com mais de setenta e cinco anos, quando recebem uma suplementação calórica durante os períodos de internação e durante a evolução ambulatorial, independente da dieta usual, apresentam nítida melhora das condições gerais, em comparação ao controle, que recebe somente a dieta usual. Esta suplementação, durante a internação, é oferecida na forma líquida, contendo cerca de $500 \mathrm{kcal} / \mathrm{d}$ e, no período ambulatorial, de $250 \mathrm{kcal} / \mathrm{d}$, contribuindo significativamente para a convalescência e a recuperação de estados carenciais dos idosos ${ }^{(22)}$.

\subsection{Resposta Imune}

Especula-se, também, que a suplementação de selênio, vitamina $\mathrm{E}$, cromo, carotenóides e ácido ascórbico poderia prevenir a menor expressão de interleucina- 2 e seu receptor, que ocorre no idoso, conseqüentemente melhorando ou prevenindo o declínio da resposta imune, dependente desses fatores ${ }^{(23)}$.

\subsection{Causas de desnutrição do idoso.}

A má nutrição que ocorre no idoso pode ser devida às alterações fisiológicas do envelhecimento, às condições sócio-econômicas, às doenças e à interação entre nutrientes e medicamentos. Assim sendo, as principais causas de má nutrição do idoso estão catalogadas na Tabela IV.

\section{Tabela IV - Causas de desnutrição no idoso}

A - Secundárias ao envelhecimento

1 - Gastrite atrófica, hipocloridria e diminuição do fator intrínseco, que pode ocorrer em $20 \%$ dos casos, resultando em má absorção de cálcio, vitamina B e ferro.

2 - Fibrose e atrofia das glândulas salivarés.

3 - Perda de dentes.

4 - Diminuição da atividade da lactase e outras dissacaridases.

5 - Atrofia de papilas gustatórias.

6 - Menor responsividade a opióides (dimorfina) e outros neuropeptídeos, podendo resultar em "anorexia da idade".

7 - Diminuição da sensibilidade de receptores associados ao controle da sede e, conseqüentemente, menor ingestão de água, hipodipsia e desidratação.

8 - Diminuição da capacidade cutânea de produção de vitamina D.

9 - Diminuição da absorção de fontes de ferro não-heme e da eritropoiese, resultando em anemia.

10 - Diminuição da absorção de micronutrientes, em especial do zinco e do cobre.

B - Alterações sócio-econômicas.

2 - Menor rendimento econômico, que, conseqüentemente, pode limitar a aquisição de fontes de nutritentes adequados.

3 - Isolamento, que pode desencorajar a ingestão de alimentos.

4 - A morte de entes queridos pode favorecer aparecimento de anorexia.

C - Doenças e outros fatores relacionados.

1 - Piora da capacidade funcional em geral, o que pode causar dificuldades na aquisição, estocagem, preparação e consumo de alimentos.

2 - Depressão associada a anorexia.

3 - Deterioração da função cognitiva, o que resulta em inabilidade para obter alimento, esquecimento ou incapacidade de se alimentar.
4 - Hospitalização prolongada recebendo oferta energética inferior às necessidades.

5 - Doenças gastrointestinais associadas a menor digestão e absorção de alimentos.

6 - Alcoolismo causando, entre outras, deficiências de tiamina, folato e magnésio.

7 - Diabete melito causando má nutrição secundária a gastroparesia, incontinência fecal e ingestão pobre de nutrientes.

8 - Caquexia cardíaca e a perda de minerais pelo uso indiscriminado de diuréticos.

9 - Neoplasias.

D - Interação droga-nutrientes (alguns exemplos).

1 - Drogas podem alterar o apetite, a absorção, o metabolismo, a utilização e a excreção de nutrientes.

2 - Minerais podem competir entre si, durante o processo absortivo, como por exemplo, zinco e ferro.

3 - Anticoagulantes e antibióticos podem induzir a deficiência de vitamina $\mathrm{K}$.

4 - Resinas ligadoras de colesterol ou óleo mineral podem induzir a má absorção de vitamina A, D, E e K.

5 - Antiácidos podem alterar a absorção de ferro, cálcio e vitamina B

6 - Trimetoprim, dilantim, barbitúricos, colestiramina e álcool podem resultar em deficiência de folato.

7 - As necessidades de piridoxina estão aumentadas com o uso de isoniazida e hidralazina.

8 - Deficiência de vitamina B pode estar associada com o uso de álcool, neomicinâ, colchicina, colestiramina e clindamicina.

9 - Dietas hiperprotéicas podem diminuir a atividade de agentes dopaminérgicos utilizados no tratamento da doença de Parkinson.

10 - Uso de dilantim pode dificultar a hidroxilação hepática de vitamina $D$. 


\subsection{Consequiências da má nutrição no idoso}

A má-nutrição do idoso resulta em sério comprometimento do estado geral do paciente. Como consequências, ocorre uma maior morbidade e mortalidade das doenças em geral. A Tabela $V$ são catalogados os principais efeitos da má-nutrição sobre a saúde do idoso ${ }^{(3)}$.

Tabela V - Conseqüências da má nutrição no idoso

A - Menor atividade física devido a hipotrofia muscular ou disfunção orgânica.

B - Piora de estados mórbidos em geral.

1 - Insuficiência cardíaca congestiva (proteína, tiamina, magnésio e potássio).

2 - Intolerância à glicose (gordura, potássio, cromo, hidratos de carbono).

3 - Osteopenia (vitamina D, cálcio, vitamina C, proteína, fósforo, sal, álcool).

4 - Distúrbios neuro-psicológicos (vitaminas do complexo B, energia).

C - Alterações farmacológicas.

1 - Diminuição da capacidade de transporte de drogas pela albumina.

2 - Menor distribuição de drogas lipossolúveis.

3 - Aumento da concentração sérica de drogas hidrossolúveis.

4 - Má absorção intestinal secundária à atrofia da mucosa intestinal.

D - Menor capacidade de cicatrização secundária a deficiência de proteína, $\mathrm{Zn}$ e vitamina $\mathrm{C}$.

E - Imunossupressão ${ }^{(24)}$.

1 - Má-nutrição associada com a maior morbidade e mortalidade que acompanha as doenças infecciosas.

2 - Menor capacidade de fagocitose e morte de bactérias. Função de linfócitos $T$ e células "natural killer" diminuídas.

\section{AVALIAÇÃONUTRICIONAL DOPACIENTE IDOSO}

A avaliação nutricional do paciente idoso deve ser feita de maneira padronizada e criteriosa. Na Tabela VI expostos os principais aspectos a serem considerados na obtenção de dados e posterior diagnóstico relacionados ao estado nutricional do idoso.

\section{INTERVENÇÃONUTRICIONAL ${ }^{(2)}$}

A partir do momento em que se decide pela intervenção nutricional, ou seja, pela utilização de nutrientes, como fármacos, visando o tratamento de doenças, devem ser seguidas as recomendações listadas na Tabela VII.

\section{Tabela VI - Avaliação clínica do idoso}

\section{A - História clínica}

Alteração recente e intencional do peso.

Cirurgia recente

Quantidade e qualidade da dieta ingerida.

Idiossincrasias.

Restrições religiosas.

Restrições iatrogênicas.

Intolerância à lactose.

Disfagia.

Estado dos dentes.

Capacidade funcional em geral.

Condições gerais de vida.

B - Exame físico.

1 - Estado mental.

- Depressão.

- Demência (vitamina $B_{12}$, folato, proteína).

2 - Pele.

- Palidez (anemia).

- Queilose, estomatite angular (vitaminas do complexo B).

- Petéquias, hemorragias (vitamina C).

- Hiperceratose perifolicular (vitamina A).

- Dermatite nasolabial e escrotal (riboflavina).

- Xerose, alopecia ("kwashiorkor"-marasmático). - Úlcera de decúbito ("kwashiorkor").

3 - Olhos.

- Hipervascularização conjuntival, blefarite (vitaminas do complexo B).

- Cegueira noturna, xerose e opacidade de córnea (vitaminaA).

4 - Orofaringe.

- Glossite e hipotrofia papilar (ferro).

- Glossite e estomatite (vitaminas do complexo B).

- Hemorragia gengival (vitamina C).

- Queda de dentes (cálcio).

- Palidez (ferro).

5 - Sistema cardiovascular.

- Insuficiência cardíaca congestiva (tiamina, marasmo, "kwashiorkor").

6 - Sistema digestivo. Hepatomegalia ("kwashiorkor").

7 - Sistema nervoso.

- Neuropatia periférica (vitaminas do complexo B, álcool).

- Atrofia e fraqueza muscular (vitamina D, marasmo, "kwashiorkor").

8 - Antropometria ${ }^{(25)}$.

- Índice de massa corpórea (peso-kg/altura²-m) com mais de $20 \%$ de deficiência é indicativo importante de alto risco nutricional.

C - Avaliação laboratorial.

1 - Estoques protéicos: albumina e transferrina.

2 - Imunidade: contagem total de linfócitos e testes cutâneos.

3 - Medula óssea: hemoglobina.

4 - Hidratação: eletrólitos, osmolaridade e urina rotina.

5 - Níveis específicos de vários nutrientes:

- Ferro, ferritina, vitamina $B_{12}$, folato, 25-hidroxi vitamina $\mathrm{D}$, cálcio, fósforo, magnésio e zinco. 


\section{Tabela VII - Intervenção nutricional no idoso}

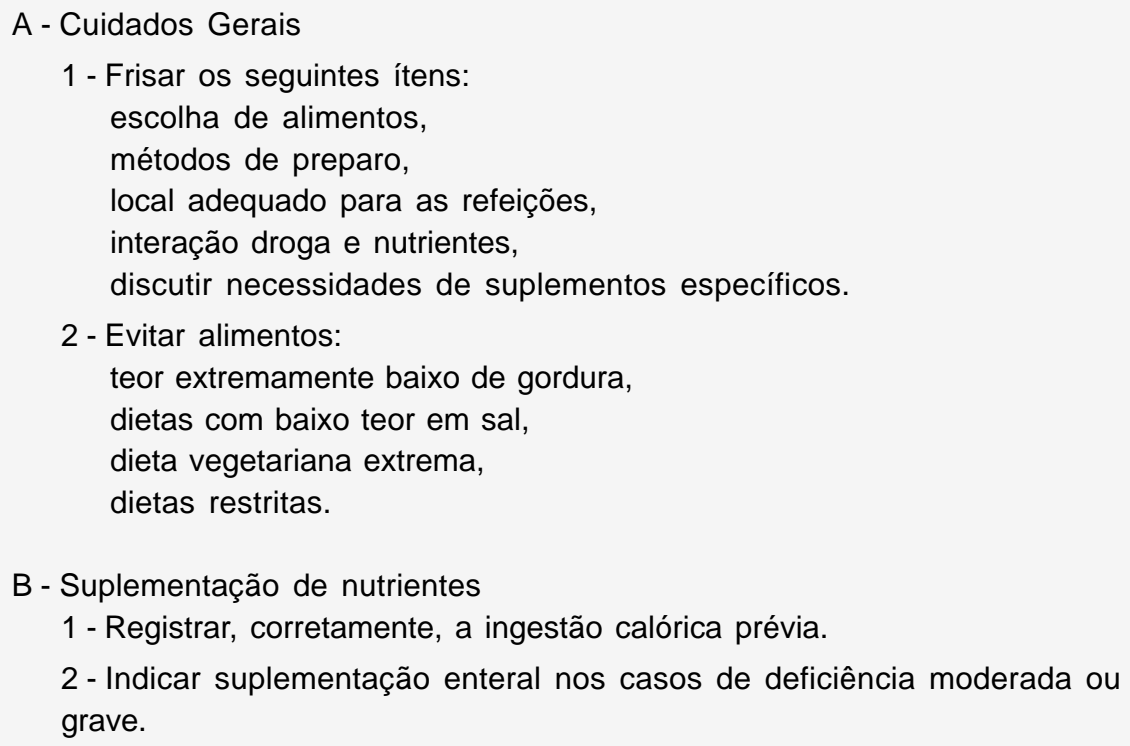

B - Suplementação de nutrientes

1 - Registrar, corretamente, a ingestão calórica prévia.

2 - Indicar suplementação enteral nos casos de deficiência moderada ou grave.

a) Usar sonda nasoduodenal de pequeno calibre e soluções livres de lactose, isosmolares e contínuas (12 a $18 \mathrm{~h} / \mathrm{dia}$ ).

b) Oferecer cerca de 35 a $40 \mathrm{kcal} / \mathrm{kg} / \mathrm{d}$.

c) Continuar oferecendo dieta via oral.

3 - Indicar nutrição parenteral, quando o trato digestivo não for utilizável (obstrução, cirurgia, íleo adinâmico, sangramento ou ressecção extensa).

C - Complicações diretamente relacionadas com o suporte.

\begin{tabular}{lcc} 
Complicação & Enteral & Parenteral \\
\hline - Intolerância psicológica & $\mathrm{x}$ & $\mathrm{x}$ \\
- Trauma ou infecção da sonda/cateter & $\mathrm{x}$ & $\mathrm{x}$ \\
- Obstrução da sonda/cateter & $\mathrm{x}$ & $\mathrm{x}$ \\
- Sangramento digestivo, diarréia & $\mathrm{x}$ & \\
- Pneumonia aspirativa & $\mathrm{x}$ & \\
- Deficiência de micronutrientes & & $\mathrm{x}$ \\
- Desequilíbrio hídrico & $\mathrm{x}$ & $\mathrm{x}$ \\
- Distúrbios de eletrólitos & $\mathrm{x}$ & $\mathrm{x}$ \\
- Doença óssea metabólica(27) & & $\mathrm{x}$ \\
\hline
\end{tabular}

A necessidades calóricas basais da pessoa com mais de sessenta anos estão, em média, diminuídas e associadas com menor atividade física. Em geral, esse grupo etário se caracteriza por menor massa protéica magra e aumento relativo da gordura corporal. Acredita-se que $30 \mathrm{kcal} / \mathrm{kg} /$ dia sejam suficientes para a manutenção dos gastos calóricos da pessoa idosa, em atividade regular. A maior parte da oferta calórica é conseguida às custas de hidratos de carbono. Recomenda-se, inclusive, que ocorra uma maior ingestão de hidratos de carbono complexos (fibras) que estariam associados à menor prevalência de constipação intestinal, câncer do cólon e diabete melito. Em contraposição, uma ingestão elevada dos hidratos de carbono, complexos pode cursar com a absorção inadequada de minerais, como, por exemplo, do zinco, cálcio, ferro e magnésio. Apesar de a quantidade de energia fornecida por $1 \mathrm{~g}$ de gordura ser igual a 9 $\mathrm{kcal}$, alimentos muito gordurosos podem não ser uma boa fonte de minerais e micronutrientes. Agordura, pre-

\section{RECOMENDAÇÕES NUTRICIONAISPARA OIDOSO}

As recomendações têm por objetivo indicar a quantidade mínima de nutrientes que seria adequada para maioria das pessoas em seu ambiente usual, sem traumas ou doenças. As recomendações para o idoso são, ainda, geralmente, extrapoladas das recomendações obtidas para crianças e adultos jovens. As situações, em que ocorre trauma orgânico (infecção, politraumatismo, cirurgia, etc) podem aumentar as necessidades de um nutriente específico na razão de 1.5 a 3 vezes o basal. ferencialmente, deve ser fornecida na forma de uma mistura de ácidos graxos saturados e insaturados. Cerca de 30-40\% da energia total, não protéica, ingerida deve ser obtida da ingestão de gordura. A ingestão de gordura torna a dieta mais palatável, o que serve para diminuir a anorexia.

A ingestão recomendada de proteína é semelhante à recomendada ao jovem adulto: $0.8 \mathrm{~g} / \mathrm{kg} / \mathrm{d}$. De maneira análoga à energia, situações clínicas como o trauma e doenças, em geral, podem estar associadas a uma maior necessidade protéica. A Tabela VIII apresenta as recomendações nutricionais para os idosos. 
Tabela VIII - Recomendações nutricionais ${ }^{(17)}$ de pessoas com mais de cinqüenta anos.

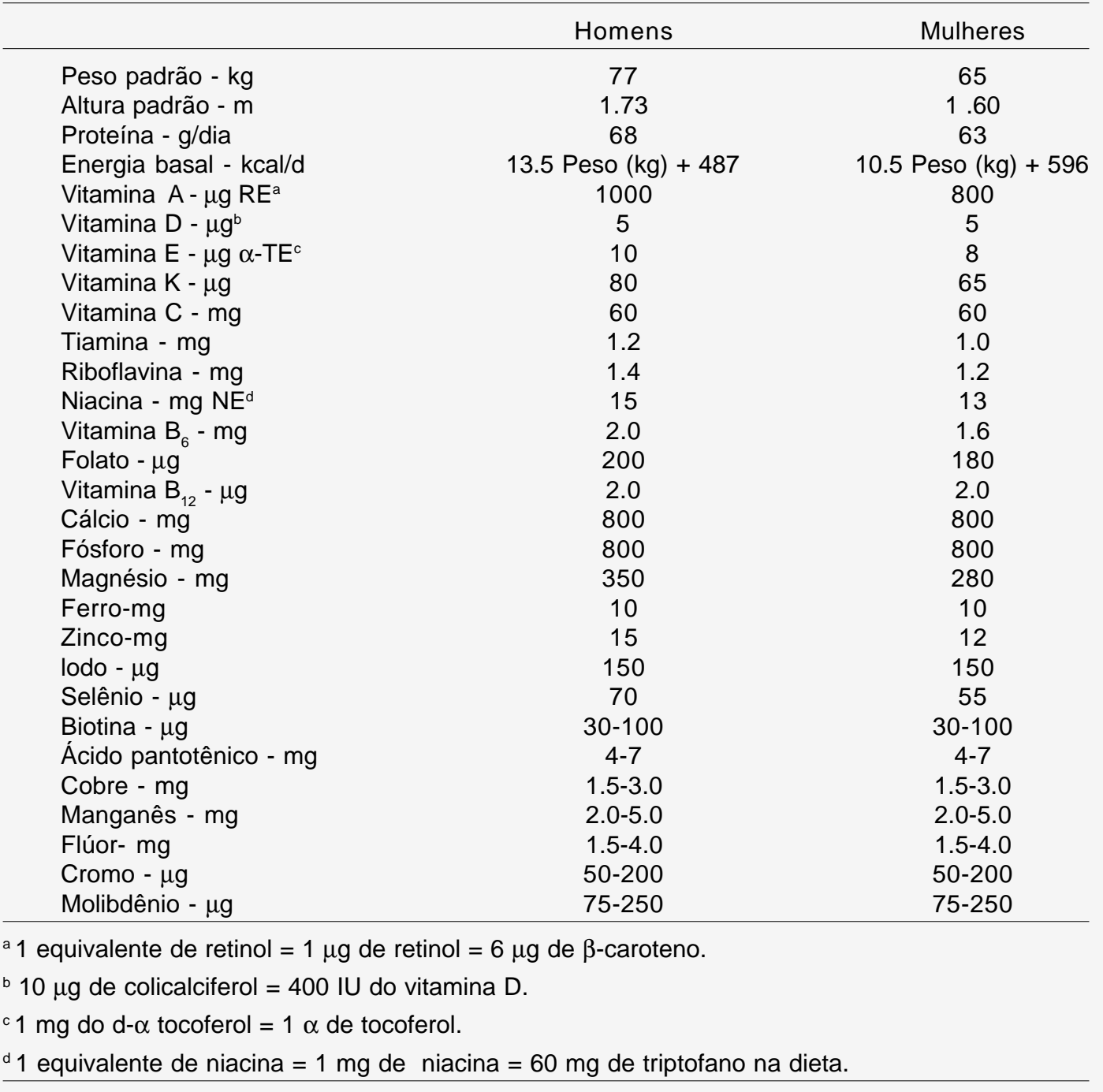

MARCHINI JS; FERRIOLI E \& MORIGUTIJC. Nutritional support in geriatric patients: definition, diagnosis, assessment and therapeutic. Medicina, Ribeirão Preto, 31: 54-61, jan./march 1998.

ABSTRACT: Increasing numbers of elderly patients are being admitted to hospital and nursing homes. Clinicians face the challenging task of developing optimal plans for individual treatment and rehabilitation for these patients. Because of the insidious nature of malnutrition in the elderly and of its mimicking of the usual aging process, it is important to recognize early warning signs of the risks of malnutrition. The physician needs to know the triggers or alerts of poor nutritional health during history/physical examination. Those in need of more comprehensive nutrition screen, which encompass not only the routine medical and dietary history and a physical exam but also a broad look at the elderly individual's psychosocial, functional, socioeconomic, and drug use aspects that are more related to malnutrition. This review paper provides a summary of the accumulated information about these matters, with the purpose of promoting active management of undernutrition of elderly people.

UNITERMOS: Nutrition. Aged. Diet Therapy. 


\section{REFERÊNCIAS}

1 - TIERNEY AJ. Undernutrition and elderly hospital patients: a review. J Adv Nurs 23: 228-236, 1996.

2 - DAVIDHIZAR R \& DUNN C. Malnutrition in the elderly. Home Health Nurse 14: 948-954, 1996.

3 - MOORE AA \& SIU AL. Screening for common problems in ambulatory elderly: clinical confirmation of a screening instrument. Am J Med 100: 438-443, 1996.

4 - WHITEHEAD C \& FINUCANE P. Malnutrition in elderly people. Aust N Z J Med 27: 68-74, 1997.

5 - MÜHLETHALER R et al. The prognostic significance of proteinenergy malnutrition in geriatric patients. Age Ageing 24: 193197, 1995.

6 - STILES NJ; BOOSALIS MG \& BOWEN J. A geriatric nutrition clinic: addressing the nutritional needs of the elderly through an interdisciplinary team. J Nutr Elder 15: 33-42, 1996.

7 - DORMENVAL V. Nutrition, general health status and oral health status is hospitalised elders. Gerodontology 12: 73-80, 1995.

8 - RISSANEN PM et al. The nutritional status of Finnish homeliving elderly people and the relationship between energy intake and chronic diseases. Age Ageing 25: 133-138, 1996.

9 - MAARAVI Y. The nutritional status of 70 year olds in Jerusalem. Isr J Med Sci 32: 620-625, 1996.

10 - REA IM; GILLEN S \& CLARKE E. Anthropometric measurements from a cross-sectional survey of community dwelling subjects aged over 90 years of age. Eur J Clin Nutr 51: 68-74, 1997.

11 - BARROCAS A et al. Nutrition assessment practical approaches. Clin Geriatr Med 11: 675-713, 1995.

12 - MOSELEY MJ. Perioperative problems: nutrition and electrolytes in the elderly. Semin Perioper Nurs 6: 21-30, 1997.

13 - INCALZI RA et al. Malnutrition in the acute care hospital: a very common problem. Ann Ital Med Int 10: 222-226, 1995.

14 - INCALZI RA et al. Nutritional assessment: a primary component of multidimensional geriatric assessment in the acute care setting. J Am Geriatr Soc 44: 166-174, 1996.

15 - MORLEY JE. Anorexia in older persons: epidemiology and optimal treatment. Drugs Aging 8: 134-155, 1996.
16 - SHEIMAN SL. Tube feeding the demented nursing home resident. J Am Geriatr Soc 44: 1268-1270, 1996.

17 -Dietary Allowances Commission and Food and Nutrition Board Recommenced dietary allowances, $9^{\text {th }}$ ed. National Academy of Sciences, National Research Council. National Academy Press, Washington, D.C., 1980

18 - VAN DER WIELEN RP et al. Nutritional status of elederly female nursing home residents; the effect of supplementation with a physiological dose of water-soluble vitamins. Eur J Clin Nutr 49: 665-674, 1995.

19 - DROR Y et al. Estimation of vitamin needs - riboflavin, vitamin $\mathrm{B}_{6}$ and ascorbic acid - according to blood parameters and functional-cognitive jand emotional indices in a selected wellestablished group of elderly in a home for the aged in Israel. $\mathbf{J}$ Am Coll Nutr 15: 481-488, 1996.

20 - BEARD JL et al. Iron nutrition in rural home bound elderly persons. J Nutr Elder 15: 3-19, 1996.

21 - BELLANTONI MF. Osteoporosis prevention and treatment. Am Fam Physician 54: 986-992, 1996.

22 - VOLKERT D et al. Nutritional support and functional status in undernourished geriatric patients during hospitalization and 6-month folow-up. Aging (Milano) 8: 386-395, 1996.

23 - MCCARTY MF. Promotion of interleukin-2 activity as a strategy for 'rejuvenating' geriatric immune function. Med Hypotheses 48: 47-54, 1997.

24 - SANDSTEAD HH et al. Zinc nutriture in the elderly in relation to taste acuity, immune response, and wound healing. Am J Clin Nutr 36: 1046-1059, 1982. Suppl.

25 - MASTER A \& LASSER R. Tables of average weight and height of Americans aged 65-94 years. JAMA 172: 658-662, 1960.

26 - SCHNEIDER EL et al. Recommended dietary allowances and the health of the elderly. N Engl J Med 314:157-160, 1986.

27 - PARFITT AM et al. Vitamin D and bone health in the elderly. Am J Clin Nutr 36:1014-1031, 1982. Suppl.

Recebido para publicaçáo em 30/01/98

Aprovado para publicaçáo em 25/02/98 